stimulate many governments to initiate measurements of existing levels of radioactive contamination, and to encourage research into the ways in which the radioactivity reaches the human body. This book contains just over one hundred papers reporting some of the findings. There are contributions from both Europe and America, with a preponderance of papers from Russia, Scandinavia and the United States.

The reader is led to wonder whether any other form of artificial contamination has been the subject of such exhaustivo research. Papers contributed to the eleven sessions of the symposium report the concentrations of radioactive isotopes in soil, fresh water and sea water, and describe their metabolism by plants and animals in all three environments. In addition to the detailed reports, there are five review papers by specialists in difforent branches of the subject.

The isotopes caesium-137 and strontium-90 receive most attention, because they are potentially the most dangerous constituents of fallout, but there are also accounts of work on isotopes such as zinc-65 and chromium-51, which are present in the waste discharged from nuclear reactors, and on iodine-131, which might be released in a reactor accident. The need to understand the biological properties of many radioisotopes gives practical application to some seemingly recondito studies, such as the one concerning the metabolism of chromium-51 by marine worms.

Several papers are devoted to studies on Eskimos and Lapps, for to these people belongs the distinction of having the highest known burdens of caesium-137. In 1965, the average content in each person was about $1 \mu c .$, compared with a typical content of about $0.03 \mu c$. in those of us who live in more temperate regions. Eskimos and Lapps acquire these relatively large amounts of activity in reindeer meat, which figures prominently in their diet. The reindeer becomes contaminated with caesium because it grazes on lichens which, in comparison with more rapidly growing plants, are particularly efficient accumulators of the isotope. There are papers from Russia, America and Scandinavia describing in dotail the passage of caesium-137 and other isotopes through this unique food-chain.

In trying to assess the seriousness of artificial radioactive fallout, it is useful to compare its contribution to the radiation background with that from the natural radioelements, and a group of papers reports work on the radium and thorium series. The hazard from these natural sources may not always be negligible. An extreme example is reported by a Russian worker, who describes an area with a natural background dose rate some three hundred times the average, and claims that burrowing animals show the ill effects of chronic exposure to ionizing radiations. Another paper in the same group discusses the observed concentration of stable lead in the environment, and serves to remind us that radioactive materials are not the only toxic substances which are widely distributed.

The editors and publishers have succeeded in issuing these proceedings with only moderate delay. The editors explain in their introduction that, to speed publication, the papers are printed as they were received, with only minor alterations. The most noticeable result of this is a serious lack of clarity in a few of the papers translated into English from other languages. In one paper, figure captions have been interchanged, and, in another, two graphs are printed in duplicate, but otherwise the book appears to be free from typographical errors. Its price will not recommend it to the individual, but it should find a place in the library of any institution where work of this nature is in progress. Not only does it contribute to our knowledge of biological mechanisms, but it is also a usoful compendium of data on levels of radioactivity in the environment in the years 1958 to 1965 .

L. BuRkinshaw

\section{GONADOTROPHIN CLUB MINUTES}

Recent Research on Gonadotrophic Hormones

Edited by E. Trevor Bell and John A. Loraine. (Proceedings of the Fifth Gonadotrophin Club Meeting, Edinburgh, 1966.) Pp. viii + 345. (Edinburgh and London: E. and S. Livingstone, Ltd., 1967.) 70s. net.

The Gonadotrophin Club, we are told, met five times during the past thirteen years, but this is the first publication of its deliberations. Thirty-one members from nineteen laboratories were selected to attend, and discussed the following topies in relation to gonadotrophins: bio-assay and immunological assay; chemistry; extraction from urine, blood and tissue; excretion; clinical applications; and biological and biochemical actions. Most space (eighty-three pages) is devoted to clinical applications; the remainder is divided almost equally among the other topics.

Three or four papers are presented on several aspects of each topic. Each group of papers is followed by a short, frank discussion. Editorial revision of the verbatim record and a logical progression through tho subject have enabled an extremely readable account of the meeting to be produced.

The papers are of a high standard, as one would expect from the list of participants, and a vast amount of information is presented. It is worth noticing, however, that there are no reports of the action of follicle stimulating hormone at a biochemical level, nor of efforts to examine the control of follicular growth and atresia. Progress in these two particular areas appears to be lagging.

Unfortunately, much of the work described at the meeting had been published previously, or appeared in print before publication of this volume, and will be known to prospective readers. Consequently, one has the impression that little really new work was discussed. This rather detracts from the interest and importance of the book.

The editors achieve a more useful purpose by providing a record of the discussion of each paper. Several papers were presented on most topics, thereby illuminating the different approaches which have been made. A careful study of both papers and discussion will be found rewarding, at least by those with some familiarity with the subject.

On balance, this volume provides a useful summary of the state of research on gonadotrophins in early 1966 , and illustrates the different opinions held in the various centres around the world. Those who need a concise, readable account of current thinking on this subject will do well to refer to this book. R. E. OAKEY

\section{NEUROTIC BACKGROUND}

Neurosis in the Ordinary Family

A Psychiatric Survey. By Anthony Ryle. (Mind and Medicine Monographs.) Pp. 156. (London: Tavistock Publications, Ltd.; Philadelphia and Toronto: J. B. Lippincott Company, 1967.) 32s. $6 d$.

ONE of the great problems of psychiatry is to discover the background of neurosis. Although in examining a patient we take a full history, we can rarely obtain other than a vague and tenuous picture of the environment behind the illness. Moreover, the facts we obtain are often distorted or insufficient and only severe neurosis in parents and grandparents strikes the informants as being worth mention. Slighter conditions are glossed over and frequently the whole background is blurred by prejudices, carelessness or stupidity. It is important, therefore, that not only neurotics but their families should undergo complete psychiatric examination. The person to perform this work successfully is more likely to be the general practitioner than the consultant. He knows the families, and may, indeed, have treated other relatives before the 